\title{
FARMACIAS GUADALAJARA: UN GRUPO ECONÓMICO NACIONAL
}

\section{GUADALAJARA DRUGTORES: A NATIONAL ECONOMIC GROUP}

Alejandro Sánchez Ruiz*, Beatriz Pérez Sánchez**

* Pasante de la Licenciatura en Economía. División Académica de Ciencias Económico Administrativas. Universidad Juárez Autónoma de Tabasco.

** Doctora en Economía. Profesora Investigadora. División Académica de Ciencias Económico Administrativas. Universidad Juárez Autónoma de Tabasco. https://orcid.org/0000-0002-9184-0579

Dirección para recibir correspondencia: beatrizperez10@hotmail.com.mx 


\title{
RESUMEN
}

En el mercado de medicamentos, la venta al consumidor final se concentra en cadenas de farmacias, tiendas de autoservicios, farmacias medianas y pequeñas regionales. Se particulariza el caso de la cadena farmacéutica: Farmacias Guadalajara, en un periodo de estudio de 1942 a 2017.

OBJETIVO: Conocer las características y estrategias competitivas, dimensión y estructura que le permitieron al Corporativo Fragua constituirse en un grupo económico y permanecer en el mercado.

MATERIAL Y MÉTODO: Los métodos empleados fueron: histórico y analítico con enfoque mixto (cuantitativo y cualitativo). Se utilizaron fuentes secundarias que involucran la revisión de estudios elaborados por otros investigadores, así como registros de ventas de las 500 Empresas más importantes de México de la Revista Expansión, Forbes, página web de la empresa e Informes y Reportes Anuales del Grupo Fragua y de la Bolsa Mexicana de Valores.

RESULTADOS: Las estrategias del grupo económico del sector servicios, se reflejan en: a) el crecimiento horizontal; b) diversificación de productos; c) adquisiciones de empresas; d) búsqueda de financiamiento; e) asociaciones y f) órganos de representación.

CONCLUSIONES: Es un grupo económico exitoso que ratifica las teorías de administración financiera y económica en torno a la aplicación de estrategias de crecimiento.

PALABRAS CLAVE: Grupo económico. Farmacias. Estrategias. Tienda especializada.

\begin{abstract}
In the medicine market, the end-user sale is carried out in chains of pharmacies, convenience stores, or small and medium regional pharmacies. One case in particular is: Guadalajara Drugstores, in the period from 1942 to 2017.
\end{abstract}

OBJECTIVE: To know the characteristics and competitive strategies, dimension and structure which led Corporativo Fragua to constitute itself into an economic group and remain present in the market. 
FARMACIAS GUADALAJARA: UN GRUPO ECONÓMICO NACIONAL

MATERIAL AND METHOD: Both quantitative and qualitative approaches were used, as well as a historic and analytical documentary revision. Secondary sources were consulted from different researchers and sales records from the $\mathbf{5 0 0}$ most important enterprises in Mexico according to data presented in Expansion and Forbes Magazines, Mexican Stock Market, enterprise web page and annual reports from Fragua Group.

RESULTS: The strategies implemented by the economic group in the service sector are as follows: a) horizontal growth; b) product diversification; c) company acquisitions; d) financing search; e) associations and f) representative bodies.

CONCLUSIONS: It is a successful economic group which proves that the economic and financial management theories are correct when referring to growth strategies implementation.

PALABRAS CLAVE: economic group, drugstores, strategies, specialized store.

\section{INTRODUCCIÓN}

La empresa es la unidad económica que produce o vende mercancías y servicios que satisfacen las necesidades de la sociedad. Tratándose de medicamentos, en el pasado existía la botica y ocupaba un lugar principal en el espacio local. La modernidad implicó una nueva forma de experimentar el mundo y surgió así un cambio de imagen: la vitrina que separaba al cliente del farmacéutico, quien vendía y recetaba los medicamentos fue sustituida por un modelo de farmacias importado de Estados Unidos, el cual ofrece los productos en anaqueles, góndolas y anaqueles en grandes espacios (Sandoval, 2011).

La industria farmacéutica evolucionó en México innovando en: nuevos productos, envases, métodos de conservación, así como en su sistema de comercialización, pasando del preparado medicinal a la medicina de patente; y no sólo se modernizaron las farmacias, sino también la forma de operar en el mercado: surgieron así grandes cadenas de farmacias, aprovecharon espacios en las tiendas de autoservicios, incrementaron sus puntos de ventas para estar más cerca del consumidor final, diversificaron la oferta de sus productos y además desplazaron parte de su competencia. 
FARMACIAS GUADALAJARA: UN GRUPO ECONÓMICO NACIONAL

Entre sus estrategias de crecimiento adoptaron la formación de grupos económicos a fin de integrarse ya sea de forma vertical u horizontal, buscando sinergias, ahorros, eficiencia, y también se asociaron en defensa de sus intereses. ¿Cómo fueron posibles tales cambios?

La modernidad es parte del devenir del desarrollo capitalista de producción y de los cambios políticos y económicos sucedidos a finales del siglo XVIII en el mundo. La modernidad se caracteriza por fenómenos de configuración histórica, tales como:

1) La industrialización, sin la cual el sector farmacéutico no habría evolucionado tan de prisa;

2) La urbanización, posibilitó la construcción de viviendas y el crecimiento físico de las áreas destinadas a las ciudades que permitieron a las personas y familias aprovechar las oportunidades de proximidad, diversidad y competencia en el mercado;

3) El desarrollo de la tecnología accede el diseño y la creación de bienes o servicios que facilitan la adaptación al medio ambiente;

4) El proceso sociológico de la racionalización, alude al proceso de ordenamiento y sistematización que busca predecir y controlar la vida del hombre, orientarlo a un estilo de vida de acuerdo a patrones funcionales de producción y consumo; entre otros, que son parte del proceso de modernización (Peña, 2012).

En el caso de México, con un capitalismo tardío, el aumento de la demanda de farmacias se identifica y subsume también el crecimiento y envejecimiento de la población que conlleva a una tendencia a la urbanización.

En términos de diagnóstico económico, y de acuerdo con datos de IMS Health, empresa de servicios de información y tecnología líder a nivel mundial que proporciona a sus clientes de la industria de la salud soluciones integrales para medir y mejorar su rendimiento (Pharmaboardroom, 2017): el consumo de medicamentos de retail en México ascendió a 118 mil 618 millones de pesos anuales hasta febrero de 2015, además muestra un crecimiento de $2.8 \%$. En este mercado, las cadenas de farmacias en conjunto registran el $62 \%$ del mercado, 16\% de autoservicios y 16\% de privadas (León, 2016). 


\section{MATERIAL Y MÉTODO}

Los métodos empleados fueron: el método histórico-crítico y el método analítico con un enfoque dinámico. Se utilizaron los enfoques de origen histórico y de estrategias corporativas y organizacionales (Concheiro, Fragoso, \& Gutiérrez, 1979). El primero da mayor peso al origen histórico y a las condiciones de surgimiento para establecer la configuración definitiva, las prácticas económicas y políticas predominantes y los patrones de desarrollo de los grupos. El segundo enfoque de las estrategias corporativas y organizacionales atiende la cambiante estructura organizativa de las grandes corporaciones modernas y su tendencia a adoptar estructuras de administración y morfologías corporativas más complejas (Cordero, Santín, \& Tirado, 1983).

Se utilizaron fuentes secundarias que involucran la revisión de estudios elaborados por otros investigadores, así como registros de ventas de las 500 Empresas más importantes de México de la Revista Expansión, Forbes, sitio web de la empresa e Informes y Reportes Anuales del Grupo Fragua y de la Bolsa Mexicana de Valores.

\section{Origen}

Farmacias Guadalajara surge en marzo de 1942 justo cuando México aceleraba su industrialización mediante el modelo de sustitución de importaciones. En consecuencia, la industria farmacéutica evolucionó pasando de dedicarse a la venta de fórmulas magistrales $u$ oficinales a realizar actividades industriales (Fragua, 2011).

Durante sus primeros años de operación, Farmacias Guadalajara logró crear paralelamente a la farmacia, una red de mayoreo, distribuyendo sus productos a otras farmacias en Jalisco y los Estados circunvecinos a través de vendedores y vehículos propios; registró 130 empleados en 1947.

A principios de los sesenta inició un programa de expansión formal con el cual se crearon nuevas sucursales e incrementó su horario de servicio las 24 horas los 365 días del año. En los años setenta, el crecimiento de la economía industrial, la alta tasa de natalidad, así como una creciente competencia permitieron que Farmacias Guadalajara incorporara mayor surtido a sus tiendas, mejor servicio, ampliara sus líneas de productos, y multiplicara el número de farmacias conforme la ciudad de Guadalajara crecía en extensión (Fragua, 2001). 
FARMACIAS GUADALAJARA: UN GRUPO ECONÓMICO NACIONAL

Entre sus estrategias se creó el concepto de SuperFarmacias, que combina la farmacia con autoservicio y la tienda de conveniencia (1989). Durante la década de los noventa y ante la apertura comercial de México al mundo, Farmacias Guadalajara aplicó un plan agresivo expansionista pasando de 31 sucursales en 1991 a 197 en el año 2000; el concepto de SuperFarmacias se vio enriquecido por la comercialización en las tiendas de frutas y verduras frescas y pan horneado en el mismo lugar (Fragua, 2018).

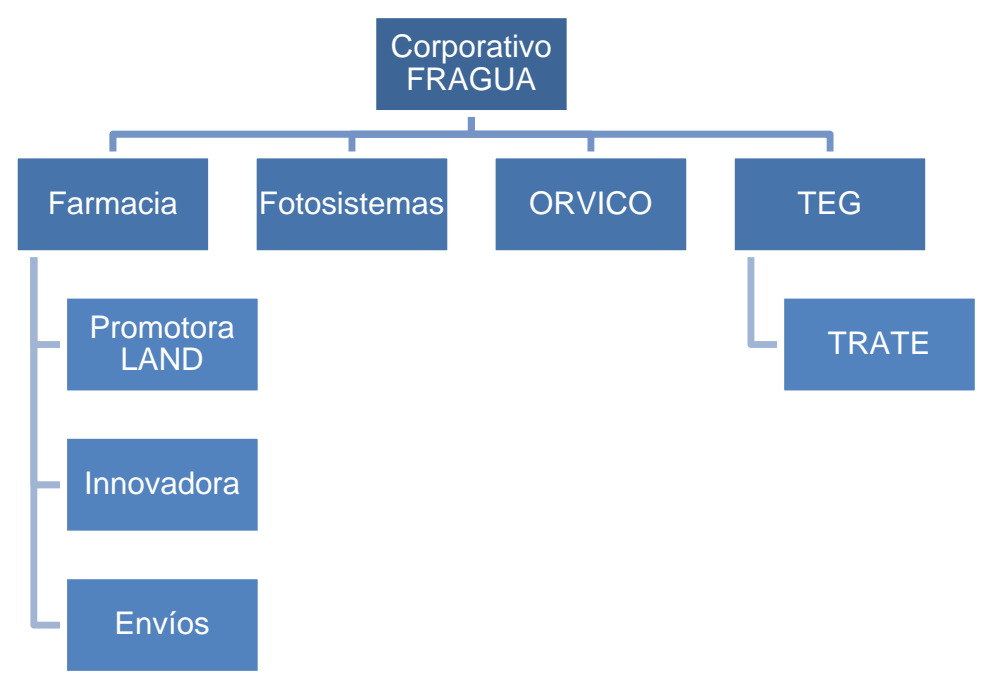

\section{Figura 1. Formación e integración del grupo económico. Estructura de Corporativo Fragua.}

Fuente: Tomado del Reporte Anual 2016 de Corporativo Fragua.

El crecimiento de Farmacias Guadalajara, trajo como consecuencia la creación de nuevas empresas que le permitieran complementar sus servicios, tales como: los de transporte y seguridad. Asimismo, diversificaron su oferta y fundaron laboratorios de revelado y tiendas de venta de artículos fotográficos. A fin de integrar este conjunto de empresas y adquirir y vender acciones de otras compañías, el 24 de septiembre de 1983 se constituyó Corporativo Fragua, S.A. DE C.V. siendo controladora de las siguientes unidades económicas:

- Farmacias Guadalajara, S.A. DE C.V. (FARMACIA), cuyo giro principal es la compraventa de productos de medicina, perfumería, fotografía, hogar, alimentos y varios.

- Fotosistemas Especializados, S.A. de C.V. (KROMI) dedicada al procesamiento y compra-venta de material de equipo fotográfico. 
- Organización de vigilancia Comercial S.A. de C.V. (ORVICO) Compañía prestadora de servicios de mantenimiento y construcción de muebles para Farmacia.

- Operadora SFG, S. de R.L. de C.V. (OPERADORA) dedicada a prestar servicios administrativos, constituida el 8 de febrero de 2005.

- Transportes y Envíos de Guadalajara, S.A. de C.V. (TEG) Sociedad dedicada a prestar servicios de carga a Farmacias Guadalajara y otras empresas del Corporativo. Cuenta con flotilla propia que permite distribuir los productos.

- En diciembre de 2005 se crea Trate de Occidente, S.A. de C.V. (TRATE), cuya función es el servicio de autotransporte en general, local y foráneo.

- Empresa Innovadora de Productos Mexicanos, S.A. de C.V. (INNOVADORA), cuyo objeto principal es la colaboración, envasado, empacado, maquila y distribución de toda clase de bebidas no alcohólicas (2011).

- El 21 de abril de 2015 se crea Promotora Land, S.A. de C.V. (PROMOTORA) empresa inmobiliaria para la adquisición de terrenos y construcciones.

- Envíos Punto a Punto, S.A. de C.V. (ENVIOS), cuyo objeto principal es la prestación de servicios de transferencia de fondos (2015) (Fragua, 2016).

- Focus 360 Publicidad, S.A. de C.V. (FOCUS) cuyo objeto es la prestación de servicios de publicidad y producción de campañas específicas (2016) (Fragua, 2017).

\section{Estrategias de expansión}

Las grandes empresas dominan el mercado por su tamaño y por su complejidad, ya que integran actividades productivas y actividades de banca y comercio permitiéndoles una gran capacidad para generar flujos monetarios que facilitan su vinculación a los mercados bursátiles. En general, las estrategias formuladas y puestas en marcha por los corporativos o grupos mexicanos son bastante completas. Incluyen diferentes combinaciones de las recetas de reestructuración que actúa en todos los niveles, financiero, tecnológico, organizacional; las nuevas formas de crecimiento por adquisición; posicionamiento o comparación con normas de excelencia internacionales; alianzas estratégicas y creación de redes; la utilización de nuevos instrumentos financieros. Las posibles combinaciones de estrategias son múltiples, dependen de la situación financiera de la empresa y de las características de su negocio principal, como estructura de costos, estrategia histórica y otros indicadores.

Los motivos más comunes para la combinación incluyen el crecimiento, los efectos sinergísticos, la obtención de fondos, mayores habilidades administrativas, consideraciones 
FARMACIAS GUADALAJARA: UN GRUPO ECONÓMICO NACIONAL

fiscales y mayor liquidez de propiedad. El Grupo Fragua aplicó estrategias centradas en el crecimiento en el territorio nacional, es decir un crecimiento horizontal: eliminando las funciones de apoyo a fin de evitar la duplicación, la compra de mercancía en mayores cantidades y la eliminación de canales de venta duplicados. Con frecuencia, una combinación horizontal de empresas aumenta las ventas al incrementar la diversidad de estilos y tamaños de los productos terminados. El crecimiento horizontal se conoce comúnmente como integración horizontal (Gitman, 1982).

Entre las estrategias del grupo Fragua se registran las siguientes:

\section{Financiamiento}

Su plan de expansión es financiado con los flujos de efectivo generados por la empresa. El 3 de octubre de 1997, corporativo Fragua realiza una oferta pública de acciones en la Bolsa Mexicana de Valores por un importe de \$230.4 millones de pesos. La empresa cotiza con la clave FRAGUAB y se clasifica como una acción de bursatilidad media (BMV, 2016).

\section{Marca propia}

A partir de 1997, Farmacias Guadalajara desarrolla su propia línea de productos que incluyen medicinas, artículos para el hogar, artículos de perfumería y alimentos, ofrece más de 1,000 productos con su marca (Fragua, 2017).

\section{Racionalidad económica}

En 2004 la empresa tomó la decisión de escindir ciertas operaciones, activos y pasivos de la empresa Farmacias Guadalajara con el objeto de constituir la central de compras de Fragua, mediante la aportación de éstos a una sociedad de nueva creación denominada SuperFarmacias Guadalajara, la cual realizaría las funciones de compras y manejo de proveedores.

Farmacias Guadalajara compra directamente a los fabricantes productos genéricos, medicamentos producidos a partir de una patente expirada, (en México ocurre a los 20 años de su autorización) cuyos efectos son iguales a los de la medicina de marca y su precio oscila entre 40 y $70 \%$ menos, con esta medida las distribuidoras mayoristas pierden cuota de mercado y la cadena gana clientes que solicitan estos medicamentos por sus bajos precios (Ilián, 2013). 
FARMACIAS GUADALAJARA: UN GRUPO ECONÓMICO NACIONAL

\section{Asociaciones bancarias}

En 2004 Farmacias Guadalajara implementó un programa de ventas que le permitió atraer a un segmento de clientes que realizan compras mediante tarjetas de crédito o débito de las instituciones bancarias Banamex y Banorte (Ramírez, 2004).

En 2011 se incrementó el servicio de corresponsalías bancarias para clientes de estos bancos, así como el pago de ciertos servicios. Estas acciones derivaron en el aumento de clientes de Farmacias Guadalajara de tal manera que en el 2016 agregaron dos nuevas instituciones bancarias a éste servicio (Expansión, 2011).

En 2016 Fragua incursionó en nuevos servicios a sus clientes mediante alianzas con instituciones financieras y de servicios; se incluyó el programa de cobros de envíos de dinero de Estados Unidos a México, de igual manera, se ofrecen servicios de giros de dinero en la República Mexicana, y corresponsalías bancarias, entre otros servicios financieros (Fragua, 2017).

De acuerdo con el reporte regulatorio R26 de la Comisión Nacional Bancaria y de Valores (CNBV), actualizado a marzo 2017, en Farmacias Guadalajara se pueden realizar operaciones de Bancomer, Banorte y American Express (depósitos, pagos de créditos y de servicios, según el banco). También existe una alianza con Western Union para las remesas (Forbes, 2017).

\section{Adquisiciones}

En 1997 el grupo Fragua adquiriere una cadena de farmacias con presencia en el centro del país, Farmacias Asa, con 14 establecimientos, mismos que después de un mes, fueron fusionadas y adecuadas al formato de SuperFarmacias (Expansión, 2011).

En 2001 adquiere la cadena de farmacias Sangre de Cristo, ubicada en el Estado de Colima, y que consta de cinco establecimientos, compra realizada por un monto de 13 mdp (López, 2009).

\section{Competir con número de farmacias}

La principal estrategia corporativa es con base en el crecimiento; es decir, la apertura de nuevas tiendas en diferentes puntos estratégicos y donde no tiene presencia. 
FARMACIAS GUADALAJARA: UN GRUPO ECONÓMICO NACIONAL

A partir del año 2000 el grupo Fragua se fija una meta expansiva que consta de inaugurar una nueva sucursal cada diez días naturales, utilizando exclusivamente recursos generados por la operación. Esa dinámica se fue repitiendo año con año, disminuyendo paulatinamente la cantidad de días naturales necesarios entre la apertura de una y otra sucursal y siendo la meta lograda y superada todos los años, comportamiento que se cumple hasta el 2008.

A partir de 2009 y hasta el 2016, se registró un promedio de inauguración de una SuperFarmacias cada cuatro días. En el año próximo pasado 2017, Farmacias Guadalajara apertura un nuevo punto de ventas cada dos días en promedio.

Tabla 1

Evolución de número de tiendas (1997-2017)

\begin{tabular}{cccc}
\hline Año & Número de tiendas & Año & Número de tiendas \\
\hline 1997 & 108 & 2008 & 651 \\
1998 & 131 & 2009 & 740 \\
1999 & 161 & 2010 & 846 \\
2000 & 197 & 2011 & 948 \\
2001 & 235 & 2012 & 1052 \\
2002 & 279 & 2013 & 1172 \\
2003 & 306 & 2014 & 1322 \\
2004 & 356 & 2015 & 1508 \\
2005 & 416 & 2016 & 1685 \\
2006 & 478 & 2017 & 1865 \\
2007 & 551 & & \\
\hline
\end{tabular}

Fuente: Elaboración propia con base en los informes anuales de Grupo Fragua.

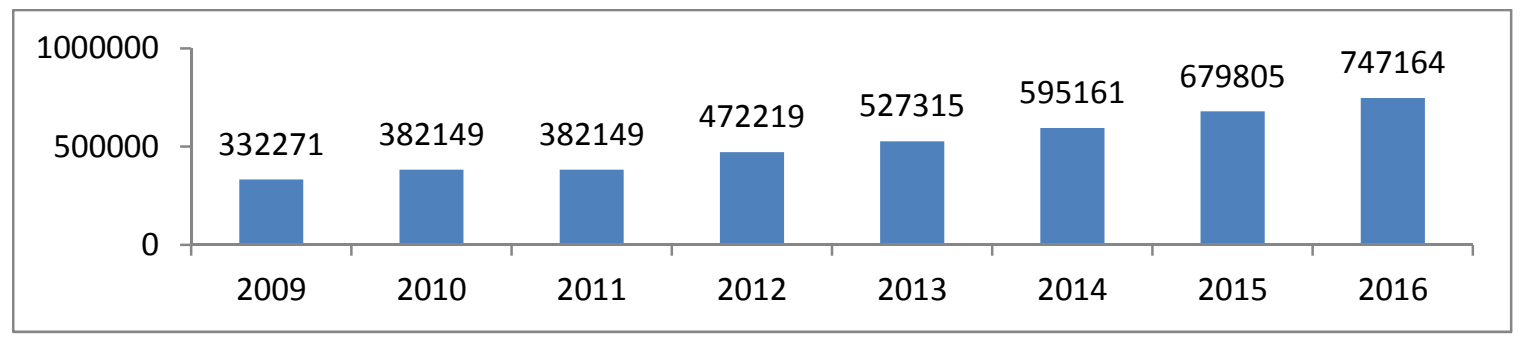

Figura 2. Área de ventas en $\mathrm{M}^{2}$ (2009-2016).

Fuente: Elaboración propia con base en los Informes anuales de Corporativo Fragua. 
En 2009 la presencia de Farmacias Guadalajara se incrementó a 177 ciudades y en 2010 registró 198 ciudades en 19 Estados de la República con 846 establecimientos. En 2011 se abrieron 102 nuevas sucursales para alcanzar los 948 puntos de ventas en 221 ciudades de 22 entidades federativas. Hacia 2012 el plan de expansión alcanzó presencia en 23 Estados y el Distrito Federal con 1052 establecimientos. Para 2013 Farmacias Guadalajara se hallaba en 271 ciudades e iniciaron operaciones 120 nuevas farmacias.

Para 2014 se integraron dentro del programa de crecimiento a 14 ciudades de 24 Estados al establecer 150 nuevas sucursales y llegar a un total de 1322 sucursales en el país. En 2015, se sumó un nuevo Estado (Tabasco) y se abrieron 186 farmacias, alcanzando los 1508 establecimientos en 325 aumentando el promedio de apertura a una nueva farmacia cada dos días.

En 2016, el Estado de Chiapas se incluyó dentro del plan expansivo con lo cual se alcanzó presencia en 352 ciudades en 26 Estados (incluyendo a la Ciudad de México) con 1685 SuperFarmacias en operación. La superficie de ventas al cierre del 2016 es de 747,164 m2 de los cuales la menor parte son propiedad de la empresa y la mayoría corresponden a locales arrendados (Fragua, 2017).

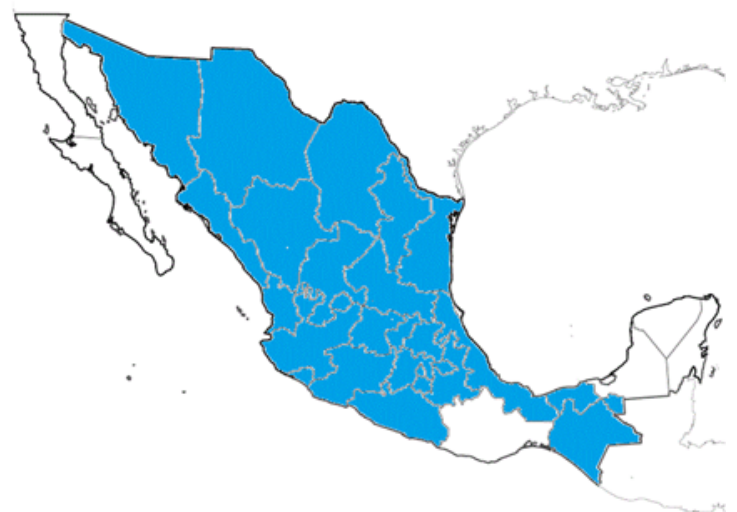

\begin{tabular}{|l|l|l|}
\hline Aguascalientes & Hidalgo & $\begin{array}{l}\text { San Luis } \\
\text { Potosí }\end{array}$ \\
\hline Chiapas & Jalisco & Tabasco \\
\hline Chihuahua & Michoacán & Tamaulipas \\
\hline Ciudad de México & Morelos & Tlaxcala \\
\hline Coahuila & Nayarit & Veracruz \\
\hline Colima & Nuevo León & Zacatecas \\
\hline Durango & Puebla & \\
\hline Edo. de México & Querétaro & \\
\hline Guanajuato & Sinaloa & \\
\hline Guerrero & Sonora & \\
\hline
\end{tabular}

Figura 3. Presencia a nivel nacional de Farmacias Guadalajara (2017).

Fuente: Elaboración propia con base en el Informe Anual 2017. 
FARMACIAS GUADALAJARA: UN GRUPO ECONÓMICO NACIONAL

\section{Distribución}

Se mantienen en coordinación los centros de distribución, la flotilla y las sucursales. Los Centros de distribución abastecen en su totalidad a los puntos de venta. Se cuenta con dos Centros de Distribución: 1) Occidente, ubicado en la zona metropolitana de Guadalajara, y 2) Noreste, asentado en la zona metropolitana de Monterrey; juntos cuentan con una capacidad de almacenaje de 80,000 m3 (Fragua, 2017).

\section{Tecnología}

En 1968 entró en la era de la computación y hoy las SuperFarmacias se encuentran conectadas en línea permitiendo un mayor control en los procesos y transacciones.

\section{Personal}

Internamente la empresa adoptó dos políticas firmes que han sido clave en el éxito de la empresa: 1) contrata hombres y mujeres jóvenes, de preparación elemental, para desarrollarlos en la empresa a través de capacitación hasta los puestos gerenciales de operación; 2) utiliza siempre tecnología de punta que optimice la interacción entre las personas y los productos manejados (Fragua, 2001).

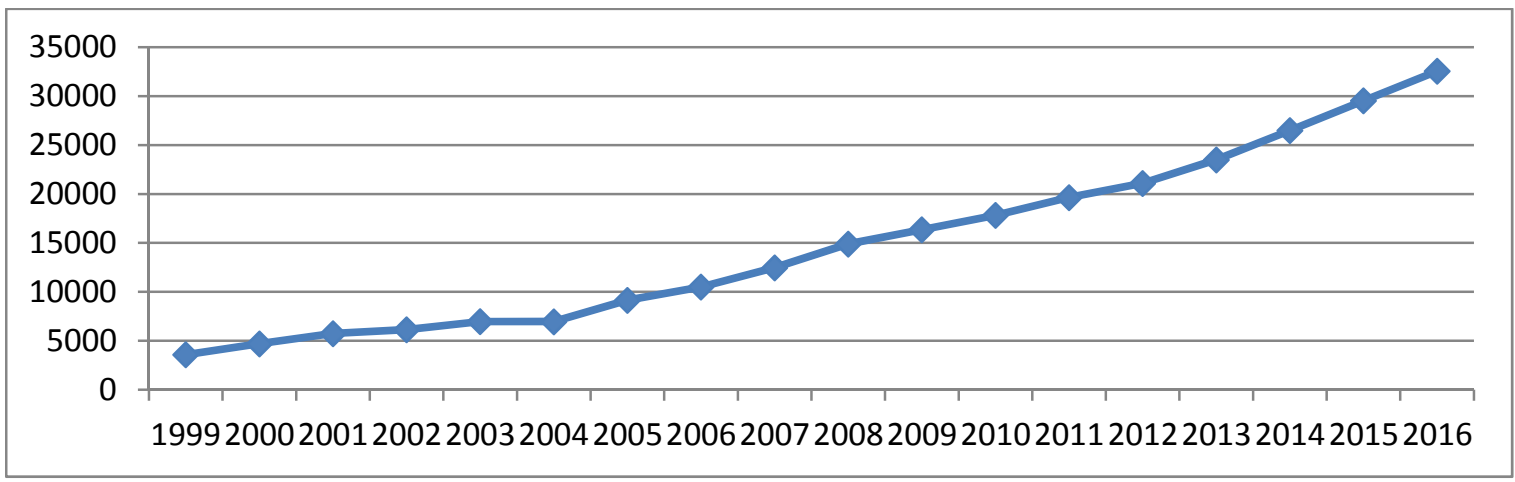

Figura 4. Evolución de número de empleados (1999-2016).

Fuente: Elaboración propia con base en el ranking anual: Las 500 empresas más importantes de México de la Revista Expansión.

En el periodo de 1999 a 2016, se observa un comportamiento de crecimiento constante en el número de empleados de la cadena de farmacias, paralelo al crecimiento en el número de 
FARMACIAS GUADALAJARA: UN GRUPO ECONÓMICO NACIONAL

tiendas y ventas, el crecimiento observado al final de la serie (32 567) corresponde a $810 \%$ del valor inicial (3577 empleados).

\section{Órganos de representación}

La cadena de Farmacias Guadalajara pertenece a la Asociación Nacional de Distribuidores de Medicinas, A.C. (ANADIM), fundada en 1944, se cuenta entre 20 empresas dedicadas a la distribución y dispensación de productos farmacéuticos, de consumo, higiene y cuidado personal en la República Mexicana (ANADIM, 2017).

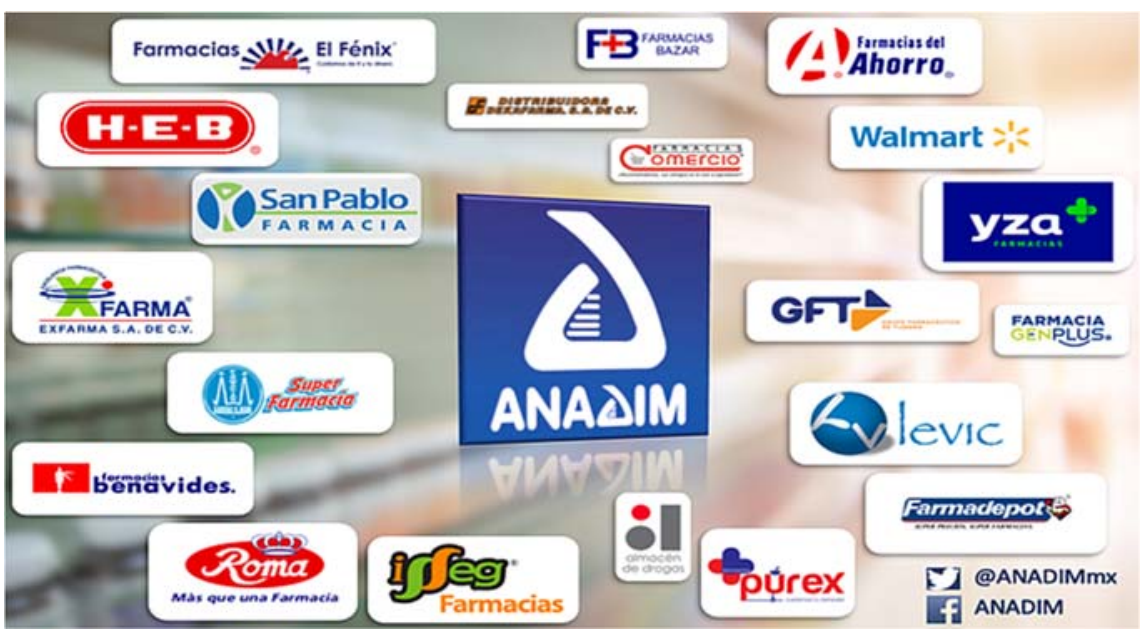

Figura 5. Empresas pertenecientes a la ANADIN.

Fuente: Tomado de (ANADIM, 2017).

La organización que representa sus intereses, promoviendo el desarrollo del comercio detallista y sus proveedores en una economía de mercado es la Asociación Nacional de Tiendas de Autoservicio y Departamentales (ANTAD), fundada en 1983, la Asociación está conformada por 104 cadenas de las cuales 32 son de autoservicio, 16 departamentales y 56 especializadas entre las que se cuentan nueve farmacias, entre ellas SuperFarmacias (ANTAD, 2017). 
FARMACIAS GUADALAJARA: UN GRUPO ECONÓMICO NACIONAL

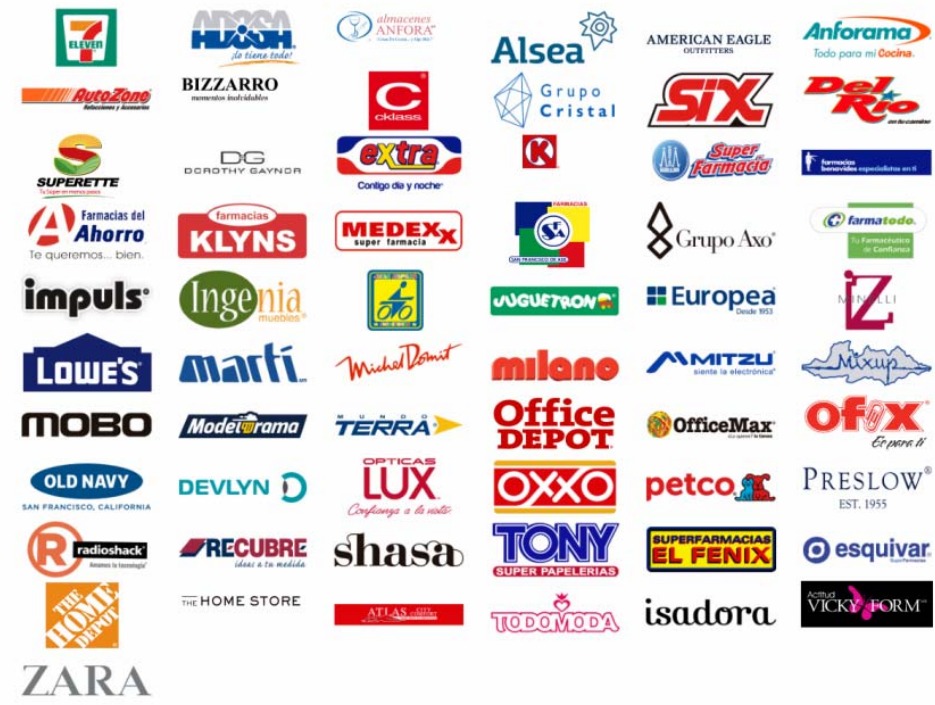

Figura 6. Tiendas Especializadas pertenecientes a la ANTAD.

Fuente: Tomado de (ANTAD, 2018).

\section{Ventas}

La cadena de SuperFarmacias de Corporativo Fragua, es una de las cadenas de farmacias más importante de México por sus ingresos, durante el periodo 1997-2017 se aprecia un comportamiento constante de crecimiento en el volumen de ventas.

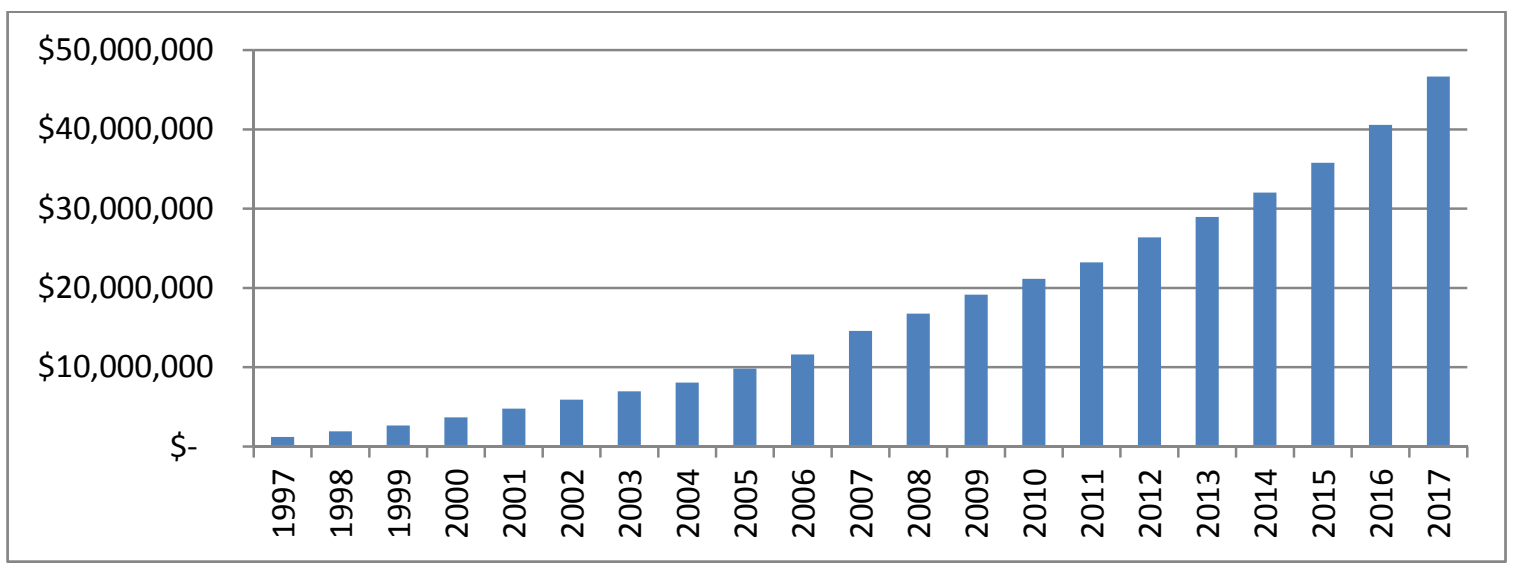

Figura 7. Ventas de Farmacias Guadalajara (1997-2017) (Millones de pesos).

Fuente: Elaboración propia con base en el ranking anual: Las 500 empresas más importantes de México de la Revista Expansión, para el año 2017 se obtuvo de (El Economista, 2018). 
FARMACIAS GUADALAJARA: UN GRUPO ECONÓMICO NACIONAL

En 2009, las ventas totales del corporativo presentaron un crecimiento de $14.2 \%$ respecto al año anterior, al transitar de 16,751.7 (en 2008) a 19,146.8 millones de pesos, en 2010 la tasa de crecimiento de ventas anual representó 10.4\%. Para 2011, el crecimiento fue de 9.8\%, en 2012 se registró un aumento en el crecimiento de las ventas que ascendió a 12.9\% y para 2013 expuso un crecimiento de $9.7 \%$.

En 2014, el volumen de ventas representó ingresos por 32,032.6 millones de pesos lo que implicó un crecimiento de 11\% respecto a 2013, en 2015 el crecimiento de ventas que se identificó es de $11.7 \%$ respecto al periodo anterior. Finalmente, hacia 2016 los ingresos por ventas fueron de 40,572.1 millones de pesos, alcanzando el nivel más, con un crecimiento de 13.3\%, en 2017 registra 46674 millones de pesos.

\section{CONCLUSIONES}

El periodo de estudio se situó de 1942 a 2017, a fin de entender el desarrollo y evolución de la empresa hasta constituir un grupo económico de capital nacional; la empresa fue evolucionando conforme se desarrolló la modernización y las políticas de industrialización que permitieron que se acelerara la industria farmacéutica, aunque más tarde que otras industrias.

El primer aspecto que llevó a la formación del Grupo Económico Farmacias Guadalajara fue la acción empresarial de dirigir las inversiones hacia la integración horizontal y diversificar su oferta a través de la creación de nuevas empresas.

La formación del Grupo Económico de Capital Nacional Fragua, ha sido el resultado de un proceso histórico que ha madurado en una estructura comercial fuertemente concentrada y centralizada.

En el contexto interno, las estrategias realizadas por Farmacias Guadalajara para crecer implicaron: a) un crecimiento horizontal en el territorio nacional; b) diversificación de productos y su propia línea de productos (1997); c) la tendencia a la integración; d) adquisiciones de empresas en 1997 y en 2001; e) asociaciones bancarias (2004, 2011, 2016, 2017); f) búsqueda de financiamiento tal como cotizar en la bolsa de valores (1997); y g) participar en asociaciones gremiales y de representación de intereses. 
FARMACIAS GUADALAJARA: UN GRUPO ECONÓMICO NACIONAL

\section{REFERENCIAS BIBLIOGRÁFICAS}

ANADIM. (2017). ANADIM. Recuperado de Asociación Nacional de Distribuidores de Medicinas, A.C. : https://www.anadim.mx/socios

ANADIM. (2017). ANADIM. Recuperado de Home: https://www.anadim.mx/

ANTAD. (2017). Asociación Nacional de Tiendas de Autoservicio y Departamentales. Recuperado de https://antad.net/la-asociacion/mision-vision-y-objetivos/

ANTAD. (2018). Asociación Nacional de Tiendas de Autoservicio y Departamentales. Recuperado de https://antad.net/especializadas/

BMV. (2016). Bolsa Mexicana de Valores. Recuperado de Empresas listadas: http://www.bmv.com.mx/es/Grupo_BMV/PerfilEmpresa/FRAGUA-5368

Concheiro, E., Fragoso, J., \& Gutiérrez, A. (1979). El poder la gran burguesía. México: Ediciones de Cultura Popular.

Cordero, S., Santín, R., \& Tirado, R. (1983). El poder empresarial en México. México: Terra Nova.

El Economista. (09 de marzo de 2018). El Economista. Recuperado de Sureste, objetivo de Farmacias Guadalajara: https://www.eleconomista.com.mx/estados/Sureste-objetivo-deFarmacias-Guadalajara-20180308-0180.html

Expansión. (20 de septiembre de 2011). Expansión. Recuperado de Farmacias Guadalajara $<$ BR> Regionales y E.

Forbes. (28 de septiembre de 2017). Forbes México. Recuperado de Fragua: Más establecimientos, menos rendimiento de capital: https://www.forbes.com.mx/fragua-masestablecimientos-menos-rendimiento-de-capital/

Fragua. (2001). Informe Anual 2000. México: Corporativo FRAGUA.

Fragua. (2011). Informe Anual 2010. México: Corporativo FRAGUA.

Fragua. (2016). Informe Anual 2015. México: Corporativo FRAGUA.

Fragua. (2017). Informe Anual 2016. México: Corporativo FRAGUA.

Fragua. (marzo de 2018). Farmacias Guadalajara. Recuperado de https://www.farmaciasguadalajara.com.mx/PaginaWebFragua/empresa/

Gitman, L. (1982). Fundamentos de administración. México: Harla.

Ilián, B. (2013). Con efectos secundarios. Expansión, pp. 123-130.

SÁNCHEZ-RUIZ A., PÉREZ-SÁNCHEZ B.

MAYO-AGOSTO 2018. Año 24, Número 69. Págs. 349-365 
FARMACIAS GUADALAJARA: UN GRUPO ECONÓMICO NACIONAL

León, G. (2016). Farmacias del Ahorro inyecta esfuerzos. Revista Mundo Ejecutivo. Edición Las 1000 empresas más importantes de México, p. 248.

López, P. (2009). Diccionario de la clase empresarial mexicana. México: UNAM.

Peña, P. (enero de 2012). Memoria, cine y modernidad: una propuesta crítica para aproximarse al pasado. Polis, pp. 115-142.

Pharmaboardroom. (diciembre de 2017). Recuperado de http://pharmaboardroom.com/pharmadirectory/ims-health-mexico/

Ramírez, Z. (17 de diciembre de 2004). El Universal. Recuperado de Bancomer y Farmacias Guadalajara firman alianza: http://archivo.eluniversal.com.mx/finanzas/43510.html

Sandoval, N. (2011). Las grandes cadenas se comen a las farmacias. Mundo Ejecutivo, p. 3641. 